\title{
UNIVERSITY FOR PEACE (UP) UNIVERSITE POUR LA PAIX
}

[Universidad para la Paz]

Ciudad Colón

Apartado 199-1250

Escazú (Costa Rica) 1980

Tel: (506) 49-1511

Fax: (506) 49-1929

Telex: 2331 macaze $\mathrm{cr}$

Founded by Resolution 35/55 of the 35th Session of the United Nations General Assembly, the activities of the University focus on: Study of Peace; Education for Peace; and Human Rights. Among its essential missions are to promote the spirit of understanding, tolerance, and peaceful coexistence among all human beings, and to contribute to the universal task of educating for peace by engaging in teaching, research, postgraduate training and dissemination of knowledge. The First Draft Medium-Term Plan (1988-1992) emphasizes: training of educators and communicators for peace, and the development of curriculums for peace education at different levels; acting as a clearing-house in providing information on peace and peace-related matters; and monitoring, analysing, and intellectually supporting the Central American peace process. The University organizes research, conferences, colloquia, meetings and workshops on peace questions. The International Peace Data and Information Centre (CEDIPAZ) ensures the functioning of the University by identifying, collection and disseminating relevant data.

Principal publication: University for Peace Newsletter (periodically, in English, Spanish).
L'Université pour la paix a été créée conformément à la Résolution 35/55 de la 35 ème session de l'Assemblée générale des Nations Unies; ses activités sontcentrées sur: l'étude de la paix; l'éducation pour la paix et les droits de l'homme. Elle a pour missions essentielles, entre autres: de promouvoir l'esprit de compréhension, la tolérance et la coexistence pacifique parmi tous les êtres humains et de participer à l'oeuvre universelle d'éducation pour la paix en consacrant ses activités principalement à l'enseignement, à la recherche, à la formation postgraduée et à la diffusion du savoir. Le Premier Projet de Plan à Moyen-Terme (1988-1992) met l'accent sur: la formation des enseignants et des responsables de la communication; le développement des programmes consacrés à l'enseignement de la paix à différents niveaux; l'action de l'Université en tant que Centre de distribution des informations sur la paix et sur les thèmes s'y rattachant. L'Université dirige, analyse et soutient moralement le processus de paix en Amérique Centrale; elle organise des programmes de recherche, des conférences, des colloques, des réunions et ateliers sur des thèmes se rapportant à la paix. Le Centre international de documentation et d'information sur la paix (CEDIPAZ) assure le fonctionnement de l'Université en identifiant, collectant et diffusant les données les plus importantes.

Publication principale: "University for Peace Newsletter" (périodiquement, en anglais et en espagnol). 